\title{
Characterization of excitonic features in self-assembled InAs/GaAs quantum dot superlattice structures via surface photovoltage spectroscopy
}

\author{
C. H. Chan \\ Department of Information Management, St. John's University, Tamsui 251, Taiwan \\ C. H. Lee and Y. S. Huang ${ }^{\text {a) }}$ \\ Department of Electronic Engineering, National Taiwan University of Science and Technology, \\ Taipei 106, Taiwan \\ J. S. Wang \\ Department of Physics and Center for Nano-Technology, Chung Yuan Christian University, \\ Chung-Li 320, Taiwan \\ H. H. Lin \\ Department of Electrical Engineering and Graduate Institute of Electronics Engineering, \\ National Taiwan University, Taipei 106, Taiwan
}

(Received 4 February 2007; accepted 14 March 2007; published online 18 May 2007)

This work systematically investigates the influence of InAs growth conditions and superlattice parameters on the optical properties of InAs/GaAs quantum dot (QD) superlattice structures grown by molecular beam epitaxy. Using surface photovoltage spectroscopy, one directly obtains the absorption spectra up to the highest confined QD levels at room temperature. Based on photoluminescence measurements at different excitation wavelengths, a feature below the fundamental transition is attributed to the transition from uncoupled dots in the bottom layers. The QD transition energy shift was found to be correlated with material intermixing, driven by enhanced strain strength with the increase of layer number and the decrease of spacer thickness, and the growth rate of InAs. A blueshift observed in low growth rate samples is indicative of greatly enhanced intermixing. For QD superlattices grown at a relatively high deposition rate of InAs, the material intermixing effect is gradually enhanced with increasing layer numbers, which will compensate the electronically coupled effect and will prevent further energy shift toward the lower energy side. The absorption wavelengths in 10- and 30-period InAs/GaAs QD superlattices with higher growth rates are near $1.32 \mu \mathrm{m}$. The results suggest that these QD superlattice structures can be used as promising active media for long-wavelength QD lasers operating at room temperature. (c) 2007 American Institute of Physics. [DOI: 10.1063/1.2733992]

\section{INTRODUCTION}

Laser structures based on quantum dots (QDs) have attracted enormous interest recently. ${ }^{1-3}$ The use of QDs as the active region in a semiconductor laser leads to an ultralow threshold current density and an extremely high thermal stability due to the spatial carrier localization effect within the QDs. Lasers with wavelengths of $1.3 \mu \mathrm{m}$, based on selfassembled InAs QDs embedded in InGaAs quantum wells, have exhibited very low threshold current. ${ }^{4}$ However, the low surface density of such QDs results in a low maximum optical gain in the QD ground state and excited states lasing at high current and/or high temperature. The operating characteristics of lasers can be readily improved by using an array of vertically coupled QDs as the active region that is sequences of QD planes separated by narrow spacer layers. ${ }^{5}$ This structural arrangement increases the total number of states in the QD ensemble. The advantages of vertically coupled QDs, besides a larger optical confinement factor, are the possibility of fast relaxation of the carriers to the ground

${ }^{a)}$ Electronic mail: ysh@mail.ntust.edu.tw state, ${ }^{6}$ a short radiative recombination time, ${ }^{7}$ and the possibility of efficient tunneling of electrons and holes between QDs within the stacked dot columns.

Previous reports on the determination of the QD energy levels have been investigated mainly through absorption/ transmission, ${ }^{8}$ photoluminescence (PL), ${ }^{9}$ photoluminescence excitation (PLE),${ }^{10}$ photoreflectance (PR), ${ }^{11}$ as well as photoelectric spectroscopy techniques such as photocurrent (PC) measurements. ${ }^{12}$ Absorption/transmission experiments are easy to interpret, but they require removal of the substrate, which is a destructive and tedious procedure. PL detects optical transitions in the QD structures by recording the radiative recombination processes. However, not all recombination processes in QDs are radiative; in such cases PL spectroscopy is unable to provide a complete picture of the system. PLE is an efficient technique, but it necessitates the use of a tunable laser and its implementation can be laborious. PC is very sensitive to the absorption of the excitation illumination. However, for PC measurements ohmic contacts with the semiconductor surfaces are required and this may modify the electronic structure of the surface layers. PR has been demonstrated as a contactless tool, which is highly sensitive to the energy levels of QDs even at room temperature. 
TABLE I. Number of deposition periods, growth parameters, and peak positions of QD1 determined from SPS $(300 \mathrm{~K})$ of the eight studied InAs/GaAs QD superlattice structures.

\begin{tabular}{|c|c|c|c|c|c|c|c|}
\hline \multicolumn{2}{|c|}{ Sample } & $\begin{array}{c}\text { Substrate } \\
\text { GaAs(001) }\end{array}$ & $\begin{array}{l}\text { Number } \\
\text { of periods }\end{array}$ & $\begin{array}{c}\text { Spacer thickness } \\
(\mathrm{nm})\end{array}$ & $\begin{array}{c}\text { Growth rate } \\
(\mathrm{ML} / \mathrm{s})\end{array}$ & $\begin{array}{l}\text { Substrate temperature } \\
\left({ }^{\circ} \mathrm{C}\right)\end{array}$ & $\begin{array}{c}\text { Position of QD1 } \\
(\mathrm{eV})\end{array}$ \\
\hline \multirow{5}{*}{ G0 } & $A$ & $n^{+}$ & 1 & $\cdots$ & 0.029 & 485 & 0.994 \\
\hline & 3 & $n^{+}$ & 30 & 30 & 0.029 & 485 & 0.995 \\
\hline & C & $n^{+}$ & 30 & 20 & 0.029 & 485 & 0.990 \\
\hline & P & $n^{+}$ & 30 & 15 & 0.029 & 485 & 1.025 \\
\hline & $E$ & $n^{+}$ & 30 & 10 & 0.029 & 485 & 1.092 \\
\hline \multirow{3}{*}{ G1 } & $\mathrm{F}$ & S.I. & 1 & $\cdots$ & 0.085 & 510 & 0.998 \\
\hline & $\mathrm{G}$ & S.I. & 10 & 15 & 0.085 & 510 & 0.942 \\
\hline & $\mathrm{H}$ & S.I. & 30 & 15 & 0.085 & 510 & 0.945 \\
\hline
\end{tabular}

Its drawback is that many parameters are necessary in the curve-fitting process to analyze the data due to changes in both the real and imaginary parts of the dielectric constant.

On the other hand, SPS is like the PR technique. It is nondestructive and contactless, but possesses several advantages over PR. Surface photovoltage spectroscopy (SPS) has been well established as a powerful technique for studying electronic states of semiconductors. ${ }^{13}$ For instance, there are no below-band-gap oscillations originating from the heavily doped substrates, and in this case PR is not useful. ${ }^{14,15}$ The SPS technique requires both photogeneration and the spatial separation of excess charge carriers. Therefore, the information about both the optical and electrical properties of the structure can be revealed in the surface photovoltage (SPV) spectrum.

In practice, it is possible to grow QD structures with many dot layers. However, complications may arise as the structural and electronic properties of InAs dots are found to be strongly dependent on the GaAs spacer thickness between adjacent dot layers, the number of deposition cycles, and the growth conditions of dot formation. Therefore, the knowledge and eventually the control of the structural and electronic coupling in QD superlattice structures are of utmost importance in terms of device applications.

To date, very little work has been done on multilayer InAs/GaAs QD structures $N \geq 10$ by using SPS. ${ }^{16,17}$ In particular, studies of the influence of the InAs growth conditions and QD superlattices structural parameters on their optical properties have not been reported in the literature yet. In this article, optical properties of InAs/GaAs QD superlattice structures grown by molecular beam epitaxy (MBE), with different layer numbers $N$ and thickness of spacer layer $d$, are systematically investigated. SPV signals from the barrier/ substrate, wetting layer (WL), ground state, and excited states of QDs have been observed and identified. The spectral shift toward either the high- or low-energy side can be changed by tuning the dot growth conditions and superlattice parameters. Several mechanisms invoked for interpreting the experimental results will be presented and discussed.

\section{EXPERIMENT}

In this study, eight QD superlattice structures, denoted as A, B, C, D, E, F, G, and $\mathrm{H}$ were synthesized and characterized. These samples were divided into two groups G0 and G1 according to the controlled geometry and growth condi- tions. Groups G0 and G1 were both grown on $n^{+}$and semiinsulating (S.I.) GaAs(001) substrates by MBE. Group G0 consists of 30 periodically arranged layers of InAs QDs with spacings $d=30,20,15$, and $10 \mathrm{~nm}$ designated as samples $\mathrm{B}$, C, D, and E, respectively. A single-layer QD structure was used as a reference sample and denoted as sample A. Group G1 consists of samples separated by a GaAs spacer thickness of $d=15 \mathrm{~nm}$ with different layer numbers $N=1,10$, and 30 designated as samples F, G, and $\mathrm{H}$, respectively. The QDs were grown in Stranski-Krastanow growth mode by depositing 2.6 monolayers (MLs) of InAs with growth rates and the substrate temperatures of $0.029 \mathrm{ML} / \mathrm{s}$ and $485^{\circ} \mathrm{C}$, and of $0.085 \mathrm{ML} / \mathrm{s}$ and $510{ }^{\circ} \mathrm{C}$ for groups $\mathrm{G} 0$ and $\mathrm{G} 1$, respectively. The formation of QDs was controlled in situ by monitoring the high-energy electron diffraction (RHEED). The QD nucleation was seen directly via the onset of a spotty RHEED pattern. The surface density of the QDs, estimated from atomic force microscopic (AFM) images, was approximated to be $2-5 \times 10^{10} \mathrm{~cm}^{-2}$. After the deposition of QDs, a $10 \mathrm{~nm}$ thick GaAs layer was deposited uninterrupted at the same temperature to cover the surface; then, the growth temperature of the remaining layers was changed to $600{ }^{\circ} \mathrm{C}$ to improve the crystalline quality. The growth conditions of the studied samples in this work are listed in Table I.

In the SPV measurements, the photovoltage was measured between the sample and a reference metal grid electrode in a capacitive manner as a function of the photon energy of the probe beam. A soft contact mode was used to enhance the photovoltage signals. The method consisted of placing a thin indium wire around the edge of the sample surface with the metal grid pressing lightly on top. The illumination system consisted of a $150 \mathrm{~W}$ quartz-halogen lamp chopped at $200 \mathrm{~Hz}$ and a grating monochromator. A beam splitter was placed in the path of the incident light. The intensity of this radiation was monitored by a pyroelectric detector and was kept constant by a stepping motor connected to a variable neutral density filter, which also was placed in the path of the incident beam. The incident light intensity was maintained at a constant level of $\sim 10^{-5} \mathrm{~W} / \mathrm{cm}^{2}$. The SPV spectrum on the metal grid was measured with a copper plate as the ground electrode, using a buffer circuit and a lock-in amplifier. All SPV data measurements reported here are carried out at room temperature.

The PL measurements were recorded by using the $\mathrm{He}-\mathrm{Ne}$ $(633 \mathrm{~nm}) / \mathrm{Nd}-$ YAG $(532 \mathrm{~nm}) / \mathrm{He}-\mathrm{Cd}(325 \mathrm{~nm})$ laser as an 


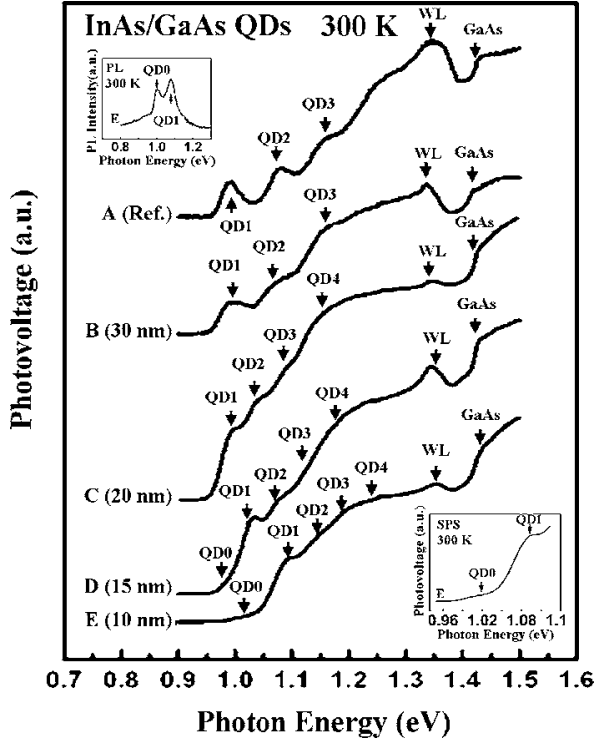

FIG. 1. Room-temperature SPV spectra of 30 layer InAs/GaAs QD superlattice structures with different spacer thicknesses (samples B-E) and the reference sample with a single QD layer (sample A). The corresponding transitions are denoted by arrows. The right-lower inset shows the magnification of the QD0 SPV spectral range for sample E in order to provide more evidence of the QD0 presence. The left-upper inset shows the PL spectrum of sample E measured at $300 \mathrm{~K}$, where the feature QD0 is clearly distinguished from QD1.

excitation source. The emitted photons were detected with a $0.5 \mathrm{~m}$ double-grating monochromator using a thermoelectrically cooled InGaAs diode. The PL measurements were taken at temperatures of 15 and $300 \mathrm{~K}$ with a power density of $\sim 5 \mathrm{~W} / \mathrm{cm}^{2}$.

\section{RESULTS AND DISCUSSION}

Figure 1 shows the SPV spectra of the reference sample (sample A) with a single QD layer and four samples B, C, D, and $\mathrm{E}$ with 30 layers of InAs/GaAs QD superlattice structures with different $d=30,20,15$, and $10 \mathrm{~nm}$ at $300 \mathrm{~K}$, respectively. In order to determine the values of QD-related transitions we have fitted the SPV data with a Gaussian line shape. ${ }^{18}$ The increase of the SPV signal at an energy near $1.34 \mathrm{eV}$ indicates the presence of a two-dimensional (2D) WL. The signal above $1.4 \mathrm{eV}$ comes from GaAs layers surrounding the $\mathrm{QD}$ and/or the substrate. At room temperature several spectral features labeled QD1-QD3 from InAs QDs are commonly visible in all samples. The ground-state transition of InAs QDs is denoted as feature QD1. The spectral features QD2, QD3, and QD4 belong to the excited states of InAs QDs. The sharp spectral features in sample A indicate that the quality is reasonably good and the distribution of dot size is relatively uniform. An additional feature QD4 at a higher energy above QD3 is detected in samples D and E. The structural coupling for thinner spacer thickness, as typically shown in Fig. 2 for sample D, complicates the strain field distribution in and around the closely stacked QDs, and has a significant modification of the band structure. The electronic coupling between QDs possibly results in the appearance of QD4. For sample B with the largest spacer thickness of $30 \mathrm{~nm}$, the peak positions of transition states are very

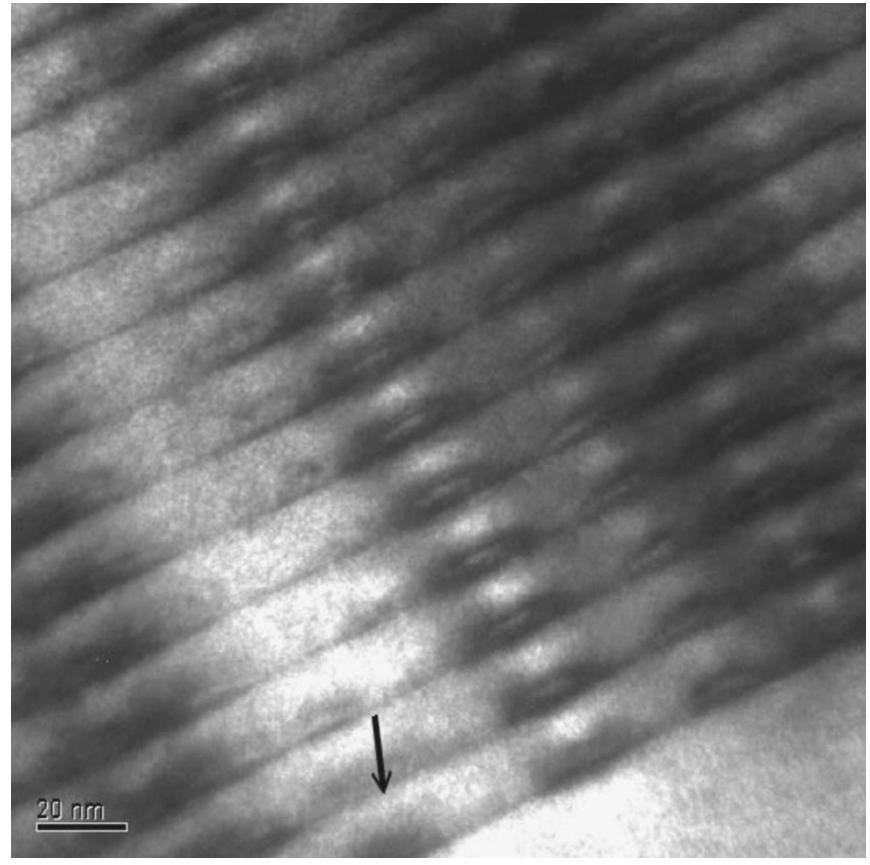

FIG. 2. The cross-sectional TEM image of sample D, with the alignment of QDs within the dot column is present. The arrow indicates an uncoupled QD in the first layer.

close to those of sample A. In this case, each QD plane behaves independently since a weak electronic coupling exists in each successive QD layer for such a thick spacer. Another observation is the larger spectral broadening of QD states in sample B. This means that the dot ensemble shows an inhomogeneous broadening of the transition states between quantum levels due to size fluctuations, even though the individual dots have zero-dimensional properties.

An unusual feature QD0 occurs at an energy below QD1 for quantum dot superlattice structures with $d=10$ and 15 $\mathrm{nm}$. A similar feature can also be found in the PL spectrum shown in the inset of Fig. 1. Note that the spectral intensity of QD0 is relative weak compared to that of QD1 in SPV spectra. Based on the comparison of the spectral intensity between QD0 and QD1, the likelihood of regarding QD0 as the fundamental transition is excluded. As is well known, the absorption strength relies not only on the overlap integral of the conduction- and valence-band envelope functions but also on the volume of the absorbing material. ${ }^{19}$ The weak intensity of QD0 leads us to designate it as arising from some uncoupled dots outside the stacked column and the fact that their density is lower than the coupled ones in the stacked columns. This feature may possibly originate from either top or bottom dot layers which have no neighbors to interact with. In order to identify which layer is most likely to cause the appearance of QD0, PL experiments with different excitation wavelengths at temperature of $15 \mathrm{~K}$ were carried out. The feature QD0 appearing in the PL spectra shown in Fig. 3 is found to be less pronounced with the excitation of the He-Cd $325 \mathrm{~nm}$ line compared with that of the He-Ne 633 $\mathrm{nm}$ line, which has a longer penetration depth. Therefore, the emission of QD0 is attributed to the uncoupled QDs in the bottom dot layers. The existence of a small amount of the uncoupled dots is also confirmed by the transmission elec- 


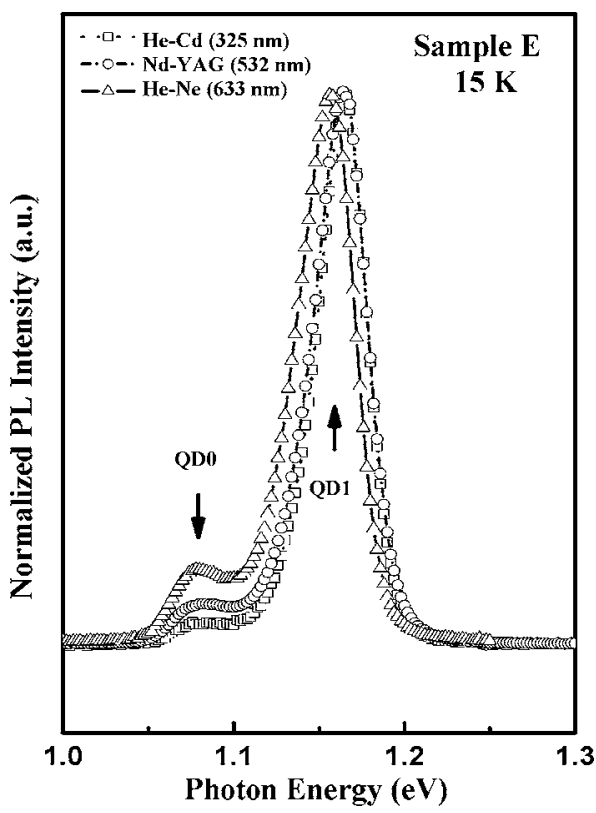

FIG. 3. The PL spectra recorded by using different excitation lines, normalized with the peak intensity of QD1 for sample E at $15 \mathrm{~K}$.

tron microscopy (TEM) image of Fig. 2, where indeed some uncoupled dots are situated near the bottom QD layers.

It is noted that as the spacer thickness is reduced below $20 \mathrm{~nm}$, the transition energies of samples D and E are considerably blueshifted with respect to those of sample B. This finding, which is inconsistent with the redshift observed by others, is explained by the increase of the dot size caused by strain field coupling ${ }^{20,21}$ and the formation of a miniband created by the electronic coupling in the vertical direction. ${ }^{22}$ Here, the probable mechanism responsible for the energetic blueshift is assigned to the enhanced strain induced intermixing occurring during the growth of the QD superlattice. Many studies showed evidence (e.g., in Ref. 23) that the dots may contain significant concentrations of $\mathrm{Ga}$ rather than being pure InAs. The increase of band gap energy arising from the incorporation of Ga composition into QDs overcompensates for the size increase, and hence results in a blueshift of transition energy.

To investigate the dependence of the energetic shift of the excitonic transitions on the growth conditions, a series of QD superlattice samples in group G1 was used for analysis. The parameters of these samples were $N=1,10$, and 30; a constant spacer thickness of $d=15 \mathrm{~nm}$ for samples $\mathrm{F}, \mathrm{G}$, and $\mathrm{H}$, respectively; a growth substrate temperature of $510^{\circ} \mathrm{C}$; and an InAs deposition rate of $0.085 \mathrm{ML} / \mathrm{s}$. In addition to signals from the 3D GaAs barrier and the 2D wetting layer, the QDs related transitions QD1-QD4, are presented in Fig. 4. As a comparison of the QD transition energies in samples $\mathrm{F}$ and A (both grown with the single dot layer), the groundstate transition of sample $\mathrm{F}$ is shifted to a higher energy by $\sim 4 \mathrm{meV}$ with respect to that of sample A. It is known that the deposition temperature has a significant effect on the total volume of material in the dots; the higher the temperature, the greater volume for a given amount of deposition. Given that the substrate temperature used during the growth for sample $\mathrm{F}$ is higher, the optical transition would shift toward

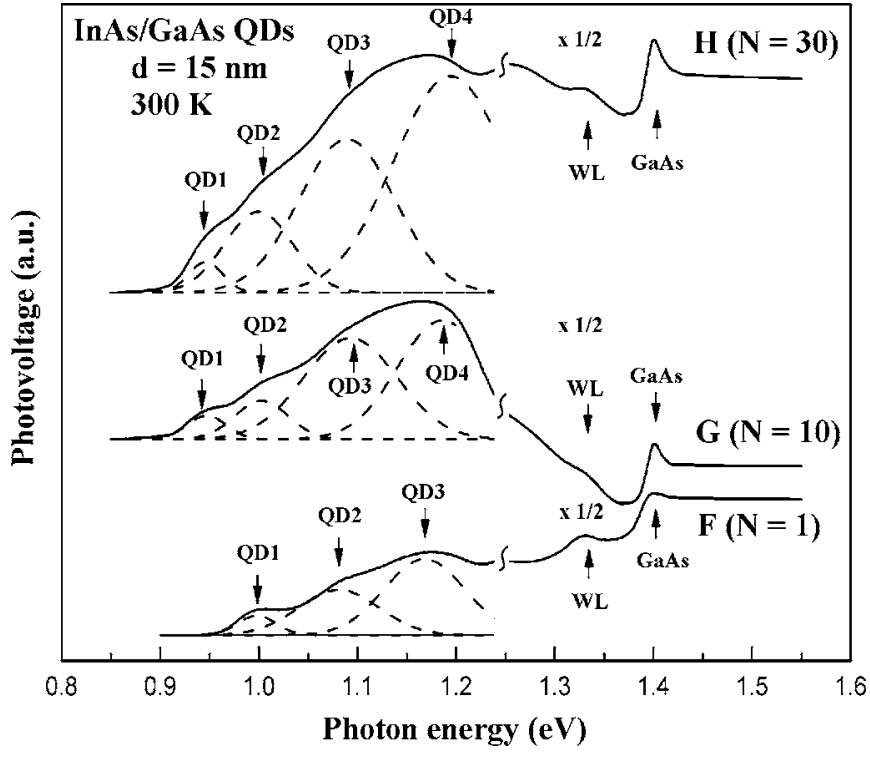

FIG. 4. Room-temperature SPV spectra of QD superlattice structures with $N=1,10$, and 30 , and a constant spacer thickness of $d=15 \mathrm{~nm}$ for samples F, $\mathrm{G}$, and $\mathrm{H}$, respectively. Arrows indicate QD related transition energies obtained from the Gaussian fitting.

the lower energy side. Obviously, this prediction is contrary to the present experimental observation, implying that the growth rate of InAs plays a dominant role in determining the energetic shift of quantum levels of QDs. Note that for samples in group G1, the spectral position of QD1 is redshifted by $\sim 56 \mathrm{meV}$ as $N$ increases from 1 to 10 and then is slightly blueshifted by $3 \mathrm{meV}$ as $N$ increases further to 30 . Increasing the number of deposition periods generally leads to a redshift of the SPV spectra due to the electronic coupling of charge carriers within the stacked dot columns. The deviation is indicative of the effect of material intermixing being gradually enhanced with increasing $N$, preventing sample $\mathrm{H}$ from further shifting the spectral position to the lower energy side. As mentioned earlier, the large strain energy may serve as a driving force for material intermixing. The resultant strain field of QDs within the stacked column is superposed by the individual field from the constituent dot. With increasing $N$ and with decreasing $d$, the accumulated strain field grows dramatically, stimulating the material intermixing to take place. Furthermore, a larger transition energy of QD1 for sample D than that for sample $H$ is observed. Both samples have the same values of $N$ and $d$ but the latter has a higher growth rate. This reveals that the effect of material intermixing in sample $\mathrm{H}$ has been suppressed in the growth process to a certain extent. Increasing the growth rate causes the formation of dots to be farther from the thermodynamic equilibrium condition, resulting in a relaxation of the intermixing. Surprisingly, the transition energy at QD1 is $0.942 \mathrm{eV}$ for sample $\mathrm{G}$ and $0.945 \mathrm{eV}$ for sample $\mathrm{H}$, corresponding to the absorption wavelengths of 1.316 and $1.312 \mu \mathrm{m}$, respectively. The result suggests that these QD superlattice structures can be used as promising active media for long-wavelength QD lasers operating at $1.3 \mu \mathrm{m}$.

\section{CONCLUSION}

Surface photovoltage spectroscopy has been used to study the influences of InAs growth conditions and superlat- 
tice parameters on the properties of InAs/GaAs QDs structures grown by MBE. SPV signals from barrier/substrate, wetting layer, ground state, and excited states of QDs have been observed and identified. Based on PL measurements at different excitation wavelengths, a feature below the fundamental transition has been attributed to optical transitions from uncoupled dots in the bottom dot layers which are less coupled to the neighbors. For samples grown at a lower InAs deposition rate, the SPV spectra show blueshifts with decreasing spacer thickness, suggesting that $\mathrm{Ga}$ incorporation in the nominally InAs has been enhanced by the strain-driven material intermixing. For samples grown at a relatively high deposition rate of InAs, the peak position of the ground state transition is redshifted by $\sim 56 \mathrm{meV}$ as $N$ increases from 1 to 10 and then is slightly blueshifted by $3 \mathrm{meV}$ as $N$ increases further to 30 . Increasing $N$ can generally lead to a redshift due to electronic coupling of charge carriers within the stacked dot columns. The deviation shows the effect of the material intermixing being gradually enhanced with the increase of $N$, compensating the electronic coupled effect and preventing a further shift toward the lower energy side. A larger transition energy of QD1 for sample D than that for sample $\mathrm{H}$ indicates that material intermixing is suppressed to a certain extent for the samples deposited with higher growth rates. The results demonstrate considerable potential of using SPS as a technique for the nondestructive characterization of self-assembled QD superlattice structures at room temperature.

\section{ACKNOWLEDGMENTS}

The authors would like to acknowledge the financial supports by the National Science Council of Taiwan under Grant Nos. NSC95-2112-M-011-001 and NSC95-2221-E011-171.

${ }^{1}$ Y. Arakawa and H. Sakaki, Appl. Phys. Lett. 40, 939 (1982).

${ }^{2}$ H. Y. Liu, B. Xu, Y. Q. Wei, D. Ding, J. J. Qian, Q. Han, J. B. Liang, and
Z. G. Wang, Appl. Phys. Lett. 79, 2868 (2001).

${ }^{3}$ J. S. Wang, R. S. Hsiao, J. F. Chen, C. S. Yang, G. Lin, C. Y. Liang, C. M. Lai, H. Y. Liu, T. W. Chi, and J. Y. Chi, IEEE Photon. Technol. Lett. 17, 1590 (2005).

${ }^{4}$ G. T. Liu, A. Stinz, H. Li, T. C. Newell, A. L. Gray, P. M. Varangis, K. T. Malloy, and L. F. Lester, IEEE J. Quantum Electron. 36, 1272 (2000).

${ }^{5}$ N. N. Ledentsov, V. M. Ustinov, V. A. Shchukin, P. S. Kop'ev, Zh. I. Alferov, and D. Bimberg, Semiconductors 32, 343 (1998).

${ }^{6}$ D. Bimberg, N. Kirstaedter, N. N. Ledentsov, Zh. I. Alferov, P. S. Kop'ev, and V. M. Ustinov, IEEE J. Quantum Electron. 3, 196 (1997).

${ }^{7}$ N. N. Ledentsov, V. A. Shchukin, M. Grundmann, N. Kirstaedter, J. Böhre, O. Schmidt, D. Bimberg, S. V. Zaitsev, V. M. Ustinov, A. E. Zhukov, P. S. Kop'ev, Zh. I. Alferov, O. A. Kosogov, S. S. Ruvimov, P. Werner, U. Gösele, and J. Heydenreich, Phys. Rev. B 54, 8743 (1996).

${ }^{8}$ T. Fromherz, W. Mac, A. Hesse, G. Bauer, C. Miesner, K. Brunner, and G. Abstreiter, Appl. Phys. Lett. 80, 2093 (2002).

${ }^{9}$ J. Y. Marzin, J. M. Gerard, A. Izraël, D. Barrier, and G. Bastard, Phys. Rev. Lett. 73, 716 (1994).

${ }^{10}$ K. H. Schmidt, G. Mederiros-Ribeiro, M. Oestreich, P. M. Petroff, and G. H. Döhler, Phys. Rev. B 54, 11346 (1996).

${ }^{11}$ C. M. Lai, F. Y. Chang, C. W. Chang, C. H. Kao, H. H. Lin, G. J. Jan, and J. Lee, Appl. Phys. Lett. 82, 3895 (2003).

${ }^{12}$ A. M. Adawi, E. A. Zibik, L. R. Wilson, A. Lemaître, J. W. Cockburn, M. S. Skolnick, M. Hopkinson, and G. Hill, Appl. Phys. Lett. 83, 602 (2003).

${ }^{13}$ L. Kronik and Y. Shapira, Surf. Interface Anal. 31, 954 (2001).

${ }^{14}$ S. Hildebrandt, M. Murtagh, R. Kuzmenko, W. Kircher, and J. Schreiber, Phys. Status Solidi A 152, 147 (1995).

${ }^{15}$ R. Kudrawiec, G. Sek, K. Ryczko, J. Misiewicz, and J. C. Harmand, Appl. Phys. Lett. 84, 3453 (2004)

${ }^{16}$ C. H. Chan, H. S. Chen, C. W. Kao, H. P. Hsu, Y. S. Huang, and J. S. Wang, Appl. Phys. Lett. 89, 022114 (2006).

${ }^{17}$ C. H. Chan, H. S. Chen, C. W. Kao, H. P. Hsu, Y. S. Huang, and J. S. Wang, J. Appl. Phys. 100, 064301 (2006).

${ }^{18}$ B. Q. Sun, Z. D. Liu, D. S. Jiang, J. Q. Wu, Z. Y. Xu, Y. Q. Wang, J. N. Wang, and W. K. Ge, Appl. Phys. Lett. 73, 2657 (1998).

${ }^{19}$ J. Toušková, E. Samochin, J. Toušek, J. Oswald, E. Hulicius, J. Pangrác, K. Melichar, and T. Šimeček, J. Appl. Phys. 91, 10103 (2002).

${ }^{20}$ G. S. Solomon, S. Komarov, J. S. Harris, and Y. Yamamoto, J. Cryst. Growth 175-176, 707 (1997).

${ }^{21}$ Z. R. Wasilewski, S. Fafard, and J. P. McCaffrey, J. Cryst. Growth 201202, 1131 (1999).

${ }^{22}$ G. S. Solomon, J. A. Trezza, A. F. Marshall, and J. S. Harris, Phys. Rev. Lett. 76, 952 (1996).

${ }^{23}$ P. B. Joyce, T. J. Krzyzewski, G. R. Bell, B. A. Joyce, and T. S. Jones, Phys. Rev. B 58, R15981 (1998). 